\title{
CONSULTA PRÉ-NATAL: SATISFAÇÃO DAS USUÁRIAS DO SUS
}

Tamara Grando 1 , Sandra Maria de Mello Cardoso², Rosane Fontana ${ }^{3}$, Francisco Carlos Pinto Rodrigues ${ }^{2}$

RESUMO: Esse estudo teve como objetivo verificar a satisfação das gestantes nas consultas de pré-natal oferecida na rede básica de um município missioneiro, situado ao noroeste do Estado do Rio Grande do Sul. Na pesquisa, de abordagem qualitativa e caráter descritivo, participaram 10 gestantes que estavam em acompanhamento pré-natal num centro municipal de atendimento à gestantes. Os dados foram coletados por meio de entrevista e a análise foi feita mediante análise do conteúdo. Nessa pesquisa foi possível perceber que as gestantes valorizam o diálogo e orientações, o que possibilita a compreensão do significado do pré-natal. Fortalecer a comunicação com as gestantes, neste período, pode favorecer o acolhimento e a qualidade do cuidado de enfermagem. PALAVRAS-CHAVE: Assistência pré-natal; Gestantes; Satisfação do usuário.

\section{PRE-NATAL CHECK-UP: UNIFIED HEALTH SYSTEM USERS' SATISFACTION}

ABSTRACT: This study aimed to check pregnant women's satisfaction with the pre-natal check-ups offered in a basic health network in a missionary town in the north-east of the state of Rio Grande do Sul. Ten mothers-to-be under pre-natal supervision at a town center for pregnancy care participated in the research, which had a qualitative approach and a descriptive character. The data was collected through interviews and analyzed through content analysis. In this research it was possible to notice that the pregnant women valued the dialog and guidance, which makes it possible to understand the meaning of the pre-natal check-up. Strengthening communication with the mothers-to-be in this period can favor the building of the relationship and the quality of nursing care.

KEYWORDS: Pre-natal care; Pregnant women; User satisfaction.

\section{CONSULTA PRENATAL: SATISFACIÓN DE LAS USUARIAS DEL SISTEMA ÚNICO DE SALUD}

RESUMEN: Ese estudio tuvo como objetivo verificar la satisfacción de las gestantes en las consultas de prenatal ofrecidas en la red básica de un municipio misionero, ubicado al noroeste del Estado de Rio Grande do Sul. En la investigación, de abordaje cualitativo y carácter descriptivo, participaron 10 gestantes que estaban en acompañamiento prenatal en un centro municipal de atendimiento a gestantes. Los datos fueron recogidos por medio de entrevista y el análisis fue hecho por medio de análisis de contenido. En esa investigación fue posible percibir que las gestantes valoran el diálogo y orientaciones, lo que posibilita la comprensión del significado del prenatal. Fortalecer la comunicación con las gestantes, en este periodo, puede favorecer la acogida y la cualidade del cuidado de enfermería.

PALABRAS CLAVE: Asistencia prenatal; Gestantes; Satisfación del usuario.

\footnotetext{
${ }^{1}$ Enfermeira. Membro do Grupo de Estudos e Pesquisas em Enfermagem Saúde e Educação da Universidade Regional Integrada do Alto Uruguai e das Missões - GEPESE URI.

${ }^{2}$ Enfermeira . Mestre em Enfermagem. Professora dos Cursos de Graduação e Pós-Graduação em Saúde da URI - Campus Santo Ângelo. Membro do GEPESE.

${ }^{3}$ Enfermeira. Doutora em Enfermagem. Professora dos Cursos de Graduação e Pós-Graduação em Enfermagem da URI - Campus Santo Ângelo. Membro do GEPESE.
}

Autor correspondente:

Sandra Maria de Mello Cardoso

Universidade Regional Integrada do Alto Uruguai e das Missões

Av. Getúlio Vargas, 1426 - 98801-570 -Santo Ângelo-RS-Brasil

E-mail: scardoso@urisan.tche.br
Recebido: $11 / 01 / 2012$ Aprovado: 02/04/2012

Cogitare Enferm. 2012 Abr/Jun; 17(2):336-41 


\section{INTRODUÇÃO}

A gestação é um fenômeno fisiológico e, por isso, seu desenvolvimento se dá, na maioria das vezes, sem complicações. Contudo, algumas gestantes podem apresentar uma evolução desfavorável, tanto para o feto, como para a mãe. A assistência ao pré-natal visa garantir um adequado andamento das gestações de baixo risco e identificar, precocemente, as pacientes que têm mais chance de apresentar uma evolução desfavorável ${ }^{(1)}$.

No que se refere à atenção com qualidade ao prénatal, o Ministério da Saúde (MS) divulgou, em 1984, o Programa de Assistência Integral à Saúde da Mulher (PAISM), que pretende, entre outras, atender a mulher na sua integralidade e em todas as fases da vida ${ }^{(2)}$. Por meio desse programa foram propostas ações que possibilitam captar precocemente as gestantes na comunidade, fazer os controles periódicos e contínuos, garantir consultas a todas as gestantes que procurarem os serviços de saúde e promover reuniões educativas. Deve o serviço dispor de área física adequada, além de equipamentos e instrumentais mínimos, assim como oferecer medicamentos básicos e suporte laboratorial, garantindo a realização dos exames complementares necessários ${ }^{(3)}$.

Sabe-se que a assistência ao pré-natal, juntamente com o cuidado, deve ser realizada integral e universalmente. Para que esse cuidado se dê de maneira satisfatória, deve-se dispor de profissionais competentes, humanos e dedicados ${ }^{(1)}$. Sendo assim, o profissional deverá atuar de forma significativa, oferecendo adequada assistência no ciclo gravídico-puerperal ${ }^{(4)}$, orientando as gestantes corretamente, a fim de minimizar ou evitar qualquer problema. É preciso desenvolver atendimento humanizado, para que se possa compreender e vivenciar com a gestante as emoções e situações da gravidez, uma vez que se trata de um momento de muitas mudanças, tanto físicas, quanto psicológicas. Cabe lembrar que nesta fase a ansiedade e os temores estão presentes, e por isso, o profissional deve oferecer um espaço à paciente para a expressão de seus sentimentos e anseios, de modo a ajudá-la a minimizá-los.

Sabendo dos inúmeros benefícios de um atendimento humanizado, o MS criou, em 2000, o Programa de Humanização no Pré-Natal e Nascimento (PHPN), com o propósito de garantir às gestantes atendimento digno e de qualidade. Como forma de acompanhar a situação de cada gestante, foi instituído o Sistema de Acompanhamento do Programa de Humanização no Pré-Natal e Nascimento (SIS Pré-Natal), o qual disponibiliza a identificação individualizada de cada gestante atendida nas Unidades que dispõem de assistência individualizada no Sistema Único de Saúde (SUS) ${ }^{(5)}$.

Além disso, o MS instituiu uma política de humanização, conhecida como Política de Humanização do Pré-Natal e Nascimento, cujo objetivo é garantir para a gestante acesso e qualidade durante o acompanhamento pré-natal ${ }^{(6)}$. Um aspecto essencial da política de humanização é o acolhimento, que deve iniciar com a recepção da mulher, quando da sua chegada na unidade de saúde, responsabilizando-se pela mesma e garantindo a ela a atenção necessária, o que implica em ouvir suas queixas, angústias e preocupações, a fim de dar continuidade ao cuidado com qualidade ${ }^{(7)}$.

Todas as gestantes, depois de acolhidas, devem ser submetidas a uma avaliação inicial, por meio de consultas de enfermagem e médica. A consulta de enfermagem, neste momento, aparece como um instrumento de extrema importância, pois garante a extensão da cobertura e melhoria da qualidade do pré-natal, por meio de ações de prevenção e promoção de saúde às gestantes $^{(3)}$.

De acordo com a Lei n. 7.498 do Exercício Profissional de Enfermagem, Decreto 94.406/87, o pré-natal de baixo risco pode ser inteiramente acompanhado pelo enfermeiro. A consulta de enfermagem é uma atividade privativa desse profissional ${ }^{(8)}$.

Buscando melhorar o cuidado de enfermagem na assistência ao pré-natal, este estudo pretende provocar uma reflexão acerca da satisfação das gestantes com o serviço de saúde. Compreendendo o que satisfaz o sujeito pode-se trabalhar para a construção de ambientes que favoreçam o cuidado solidário e humanizado. A satisfação pode ser definida como contentamento ou alegria ${ }^{(9)}$ em relação a um serviço prestado ou benefício recebido. Desta forma, é possível proporcionar um espaço mais adequado às gestantes, para que possam sentir-se mais seguras e confiantes durante as consultas.

Esse estudo teve como objetivo verificar a satisfação das gestantes nas consultas de pré-natal, oferecidas na rede básica de um determinado município da região missioneira, situado ao noroeste do Estado do Rio Grande do Sul.

\section{MÉTODO}

A pesquisa é de abordagem qualitativa e de caráter descritivo ${ }^{(9)}$. Participaram 10 gestantes que estavam em acompanhamento pré-natal num centro de atendimento às gestantes; definiu-se a amostra pelo critério de saturação dos dados ${ }^{(10)}$. Foram convidadas a participar as gestantes 
que estivessem aguardando para a consulta pré-natal de rotina em um centro de atendimento às gestantes. Foram critérios de inclusão na pesquisa: estar em acompanhamento pré-natal no turno da manhã, aceitar e formalizar a participação no estudo.

Optou-se por desenvolver a pesquisa neste Centro de Atendimento às Gestantes, por ser capaz de retratar o cotidiano do trabalho da equipe de saúde acerca da temática em estudo, como também por tratar-se de um núcleo capacitado para esse tipo de atendimento. O Centro possui quatro médicos obstetras, distribuídos nos turnos da manhã e da tarde e uma enfermeira responsável pelo setor. Não são realizadas consultas de Enfermagem por esta profissional, sendo que essa atividade somente é realizada em meados do segundo semestre de cada ano, quando os acadêmicos do sexto semestre de enfermagem de uma universidade local realizam o estágio supervisionado na disciplina de Saúde da Mulher. Nesta ocasião é que a pesquisa foi realizada

Os dados foram coletados por meio de uma entrevista na qual foi utilizado instrumento semiestruturado. A análise dos dados foi feita mediante análise do conteúdo das falas dos sujeitos; os dados empíricos foram organizados e submetidos à leitura e re-leitura visando à obtenção dos núcleos de sentido, extraindose as categorias temáticas ${ }^{(10)}$.

Para o desenvolvimento desta pesquisa foram respeitadas as diretrizes e normas regulamentadoras de pesquisa com seres humanos, da Resolução 196/96 do Conselho Nacional de Saúde ${ }^{(11)}$. A coleta de dados se desenvolveu, após aprovação do Comitê de Ética da Universidade Regional Integrada do Alto Uruguai e das Missões (URI), Campus de Santo Ângelo, sob protocolo n. 0090-4/PPH/10. Foi apresentado aos sujeitos da pesquisa, antes de participarem do estudo, um Termo Consentimento Livre e Esclarecido para que assinassem, e também foram informados do objetivo do estudo, bem como do direito a participar ou não da pesquisa, e livre decisão de desistir se assim o desejassem. Para que fosse mantido o anonimato dos sujeitos, eles foram identificados por nomes de flores.

\section{RESULTADOS}

A idade das entrevistadas variou de 19 a 37 anos. Grande parte delas era casada, sendo que maioria já havia tido gestações anteriores. O grau de escolaridade predominante foi o ensino médio completo e a maior parte das entrevistadas possuía a renda de um salário mínimo.
Os dados empíricos foram organizados e submetidos à leitura e re-leitura visando à obtenção dos núcleos de sentido, extraindo-se três categorias temáticas: $A$ consulta de enfermagem atribuindo satisfações às usuárias, A satisfação advinda do acolhimento e $O s$ procedimentos e exames laboratoriais e a atuação da enfermagem como fonte de satisfação.

\section{DISCUSSÃO}

\section{A consulta de enfermagem atribuindo satisfações às usuárias}

Percebeu-se que a consulta de enfermagem é significativa no processo de cuidado à gestante, como se pode observar na seguinte fala:

Não considero satisfatórias as consultas neste centro de atendimento, porque as consultas médicas são muito rápidas, não há muito diálogo com o médico. Já as consultas de enfermagem, realizadas pelos acadêmicos, são mais satisfatórias. (Crisântemo)

A partir desse depoimento, pode-se afirmar que as consultas de enfermagem representam um espaço de comunicação com as gestantes, visto que promovem o diálogo com as usuárias, permitindo a expressão de experiências, sentimentos, anseios e resolução de dúvidas advindas desta fase. Além disso, a escuta e o diálogo facilita o vínculo entre a gestante e a enfermeira, o que é determinante para que haja um atendimento humanizado.

A consulta de enfermagem no pré-natal consiste no acompanhamento da gestante, servindo como um momento de aprendizagem para a mulher e sua família, permitindo ainda detectar anormalidades com a mãe e a criança ${ }^{(12)}$. Além disso, é um instrumento básico para redução da mortalidade materna e infantil, além de ser uma ferramenta importante tanto para ampliar a cobertura pré-natal como para aumentar a qualidade dessa assistência, por meio de ações de prevenção e promoção à saúde das gestantes ${ }^{(3)}$, tornando-se imprescindível para que haja um adequado acompanhamento do binômio mãe-filho durante o período de gestação ${ }^{(13)}$.

\section{A Satisfação advinda do Acolhimento}

O acolhimento é um elemento essencial no atendimento. A forma como o profissional atende a gestante, o cuidado ao falar, a linguagem simples e compreen- 
sível, a sensibilidade e a capacidade de percepção dos anseios são condições essenciais para que elas retornem ao serviço. Sendo assim, a qualidade na assistência de saúde, atendendo as reais necessidades da população mediante preparo e qualificação dos profissionais da saúde ${ }^{(14)}$, são fatores que facilitam o acolhimento, permitindo que as pacientes recebam atendimento que corresponda às suas necessidades, como se percebe nas seguintes falas:

Nas consultas de enfermagem, são realizados os procedimentos (peso, ausculta dos batimentos fetais e medida da altura uterina), e há um bom acolhimento, tanto pela enfermeira, quanto pelo médico. (Rosa)

As consultas com o médico são mais rápidas ele apenas escuta os batimentos do bebê. As consultas de enfermagem são mais longas, o acolhimento é melhor, há verificação do peso, da pressão, medem a altura uterina e também escutam os batimentos do coração do bebê. (Lírio)

Percebe-se que, para as gestantes que realizam o pré-natal neste Centro de Atendimento, o acolhimento tem sido bom por parte da equipe, em especial por parte dos profissionais enfermeiros. Além disso, tem correspondido às suas expectativas no que se refere às orientações recebidas e demais procedimentos realizados. Além da valorização do diálogo pelas pacientes deste serviço, há a valorização dos procedimentos; ambas ferramentas importantes em uma consulta.

O acolhimento às usuárias é um instrumento que deve ser utilizado em virtude das possibilidades de efetivação do cuidado humanizado, uma vez que preconiza o encontro, o diálogo, a escuta, o vínculo e o respeito às diferenças existentes entre a usuária e os profissionais de saúde ${ }^{(15)}$.

Um serviço deve ser sensível e acessível para que ocorra um bom acolhimento ${ }^{(16)}$. A avaliação, pelas usuárias, quanto aos serviços prestados, em função de suas necessidades e expectativas ${ }^{(17)}$, é determinante para assegurar um atendimento de qualidade. Neste sentido, embora os relatos das gestantes evidenciem a prática do acolhimento pelos profissionais, ainda há muito a ser melhorado, pois um atendimento de qualidade acontece quando há interação entre a equipe, no qual se utilizem mecanismos como a transdisciplinariedade, em que os saberes se complementem, visando a excelência do cuidado.

\section{Os procedimentos e exames laboratoriais e a atua- ção da enfermagem como fonte de satisfação}

Um atendimento de qualidade tem como base o fortalecimento da assistência que é prestada à paciente, além de desenvolver um papel fundamental no quesito satisfação. Nesse sentido é dever do profissional que atende às gestantes esclarecer quanto à importância do pré-natal, bem como da realização dos exames laboratoriais e procedimentos realizados em cada consulta.

Dentre os procedimentos que devem ser realizados estão a ausculta dos batimentos cardiofetais, medida da altura uterina, peso e verificação de pressão arterial, entre outros. Porém, algumas das gestantes entrevistadas relataram que determinados procedimentos são apenas realizados pela enfermeira, como podemos perceber nas citações a seguir:

A enfermeira verifica o peso, a pressão arterial e também mede a altura uterina, nas consultas com o médico nenhum desses procedimentos foi realizado. (Copo de Leite)

Nas consultas de enfermagem é verificado o peso, a pressão e também recebo diversas orientações, por parte do médico, recebo somente as orientações. (Cravo)

Os procedimentos realizados em uma consulta pré-natal são importantes para que se possa detectar precocemente qualquer alteração, a fim de tratar adequadamente e, se necessário, encaminhar para um nível de assistência mais complexo. Nessa pesquisa foi possível perceber que as gestantes valorizam esses procedimentos:

Os procedimentos somente a enfermeira que realiza, o médico não realiza nenhum dos procedimentos. (Margarida)

É verificado o peso e pressão, medem a altura uterina, mas somente a enfermagem que realiza esses procedimentos. (Tulipa)

Essa valorização das gestantes está ligada à qualidade da oferta de um serviço e cabe aos profissionais da saúde oferecer um pré-natal de qualidade. Para que isso possa acontecer, é importante que os mesmos sejam comprometidos e dedicados, além de competentes para 
identificar os problemas que eventualmente possam ocorrer, e de modo a minimizar ou evitar sequelas.

Nesse processo, é fundamental atentar para as mudanças físicas e psicológicas típicas desta fase e que podem ser detectadas pelo exame físico e realização de alguns procedimentos. O enfermeiro deve estar preparado cientificamente para corresponder a essa expectativa que as usuárias demonstram nas falas e que as satisfazem e atualizar-se no intuito de qualificar o cuidado. $\mathrm{O}$ enfermeiro possui embasamento teórico-científico e respaldo legal para prestar assistência ao pré-natal de baixo risco e, é esperado dele o acompanhamento e a assistência às gestantes ${ }^{(18)}$.

De acordo com a Lei n. 7.498 de 25 de julho de 1986, a qual dispõe sobre a regulamentação do exercício de Enfermagem, cabe ao enfermeiro realizar a consulta e a prescrição da assistência de enfermagem; prescrever medicamentos e solicitar exames, como elemento componente da equipe de saúde e desde que estabelecidos em Programas de Saúde Pública e em rotina aprovada pela instituição; além de prestar assistência às gestantes, parturientes e puérperas e realizar atividades educativas em saúde ${ }^{(18)}$.

Ao esclarecer as pacientes quanto à importância e motivo da realização dos exames laboratoriais solicitados, a maioria das entrevistadas declarou que os profissionais que as atendem nas consultas de pré-natal dão esclarecimentos a respeito dos exames solicitados, bem como da sua devida importância. Algumas relataram não receber essas informações, e uma delas declarou receber esclarecimento apenas por parte da enfermagem.

Com os exames laboratoriais realizados no prénatal, é possível identificar e reduzir muitos problemas de saúde que costumam atingir a mãe e seu bebê. Além disso, estão disponíveis, gratuitamente, na rede pública e é fundamental que todos esses dados sejam registrados na carteira de gestante. Esta funciona como elo na assistência hospitalar e na unidade básica, bem como em um prontuário na unidade de saúde.

Os exames laboratoriais realizados, em toda a rede pública, de acordo com o MS são: hemograma completo, na avaliação da anemia e de possíveis infecções; glicemia, pesquisando a existência de diabetes; tipagem sanguínea; exame de urina-EAS (elementos anormais do sedimento) ou urina tipo I na detecção de infecção urinária e urinocultura na identificação da bactéria causadora da infecção, assim como a que tipo de antibiótico ela se mostra sensível. Ainda, parasitológico de fezes; sorologia para sífilis (VDRL, TPHA) que, se positiva, a gestante deverá ser tratada com penicilina, podendo afastar o risco de transmissão; sorologia para toxoplasmose; sorologia para rubéola; sorologia para hepatite B; Elisa - AntiHIV ${ }^{(6)}$.

Com todos esses exames disponíveis gratuitamente na rede pública, é possível diminuir a mortalidade materna. Mas, essa possibilidade existe se a gestante entender a importância de realizar o pré-natal, bem como fazer esses exames. Para que isso aconteça é imprescindível que os profissionais da saúde acolham as gestantes, prestem um serviço humanizado e de qualidade. Nisso se inclui o fornecimento de informações sobre o significado e a importância da realização de exames e dos procedimentos técnicos.

\section{CONSIDERAÇÕES FINAIS}

A gestação constitui-se num fenômeno fisiológico que envolve muitas mudanças biológicas, psicológicas e culturais. Isto leva-nos a entender que os cuidados no pré-natal devem ultrapassar a questão biológica e tecnicista de atenção às usuárias. Desta forma, a assistência nas consultas de pré-natal deve propiciar um adequado andamento das gestações, de modo que esta ocorra de forma saudável.

Nessa pesquisa foi possível perceber que as gestantes valorizam o diálogo e orientações, o acolhimento e os procedimentos e julgam a enfermagem como profissionais atentos às tecnologias sejam leves ou duras.

A comunicação entre as mulheres no período gravídico e os profissionais de enfermagem que as atenderam durante as consultas de pré-natal mostrou-se positiva na satisfação das gestantes. Pode-se perceber que a consulta de enfermagem contribui para que elas enfrentem este período com mais tranquilidade e confiança, pois esse momento lhes permite expressar suas dúvidas, medos e anseios comuns nesta etapa da vida, além de fornecer esclarecimentos no que se refere aos procedimentos e exames solicitados. Deste modo, foi possível formar uma relação de confiança e satisfação da gestante com o serviço de saúde.

Porém, para que a assistência de enfermagem realmente seja de qualidade, torna-se necessário que as consultas de enfermagem estejam disponíveis nos 12 meses do ano, já que essas só acontecem no período de agosto a novembro, durante o estágio supervisionado de Saúde da Mulher.

Sugerimos reflexões por parte dos gestores e profissionais desta unidade de atenção à gestante para que essas atividades tenham continuidade e que recursos humanos sejam alocados a fim de que as necessidades destas usuárias sejam amplamente respeitadas, considerando-se que 
o acesso e o acolhimento deve ser uma prioridade de um sistema resolutivo e promotor da saúde pública.

\section{REFERÊNCIAS}

1. Freitas F, Martins-Costa SH, Ramos JGL, Magalhães JA. Rotinas em Obstetrícia. $5^{\mathrm{a}}$ ed. Porto Alegre:Artmed; 2006.

2. Ministério da Saúde (BR). Assistência integral à saúde da mulher: bases de ação programática. Brasília; 1984.

3. Shimizu HH, Lima MG de. As dimensões do cuidado pré-natal na consulta de enfermagem. Rev Bras Enferm. 2009;62(3):387-92.

4. Dourado VG, Pelloso SM. Gravidez de alto risco: o desejo e a programação de uma gestação. Acta Paul. Enferm. 2007;20(1):69-74.

5. Moimaz SAS, Garbin CAS, Garbin AJI, Zina LG, Yarid SD, Soares FKM et al. Sistema de informação pré-natal: análise crítica de registros em um município paulista. Rev Bras Enferm. 2010;63(3):385-90.

6. Ministério da Saúde (BR). Secretaria de Políticas de Saúde. Programa de humanização no pré-natal e nascimento. Brasília, 2000.

7. Ministério da Saúde (BR). Manual Técnico Pré-Natal e Puerpério Atenção Qualificada e Humanizada. Caderno n 5. Brasília; 2006.

8. Conselho Federal de Enfermagem. Decreto n. 94.406 de 08 de junho de 1987: regulamenta a Lei n. 7.498, de 25 de junho de 1986, a qual dispõe sobre a regulamentação do exercício da enfermagem. Brasília (Brasil): COFEN; 1987.

9. Ferreira ABH. Minidicionário da língua portuguesa. $3^{\mathrm{a}}$ ed. Rio de Janeiro: Nova Fronteira; 1993.

10. Minayo MCS. Ciência, técnica e arte: desafio da pesquisa social. Pesquisa social: teoria, método e criatividade. $21^{\mathrm{a}}$ ed. Rio de Janeiro:Vozes; 2004.

11. Ministério da Saúde (BR). Conselho Nacional de Saúde. Diretrizes e normas regulamentadoras da pesquisa envolvendo seres humanos. Resolução n.196, de 10 de outubro de 1996. Brasília; 1996.

12. Araujo SM, Silva MED, Moraes RC, Alves DS. A importância do pré-natal e a assistência de enfermagem. Veredas FAVIP. Rev. eletr. ciências. 2010;3(2):61-7.

13. Silveira MAM, Lucena NBF, Guerreiro EM, Rodrigues
DP. Qualidade da assistência pré-natal: concepções de enfermeiros e gestantes. In: $61^{\circ}$ Congresso Brasileiro de Enfermagem; 2009 Dez. p. 1-3; Fortaleza, Brasil. Fortaleza:ABEn; 2009.

14. Benigna M J C, Nascimento W G, Martins JP. Pré-natal no programa saúde da família (PSF): com a palavra, os enfermeiros. Cogitare enferm. 2004;11(1):39-43.

15. Martins JJ, Nascimento ERP, Geremias CK, Schneider DG, Schweitzer G, Mattioli NH. O acolhimento à família na unidade de terapia intensiva: conhecimento de uma equipe multiprofissional. Rev Eletr. Enf. [Internet] 2008;10(4):1091-101.

16. Pinho LB, Hernández AMB, Kantorski LP. O discurso sobre o acolhimento e a acessibilidade nos serviços comunitários de saúde mental. Cogitare enferm. 2009;14(4):612-9.

17. Lima MDS, Ramos DD, Rosa RB, Nauderer TM, Davis R. Acesso e acolhimento em unidades de saúde na visão dos usuários. Acta Paul. Enferm. 2007;20(1):12-7.

18. Cunha MA, Dotto LMG, Mamede MV, Mamede FV. Assistência pré-natal: competências essenciais desempenhadas por enfermeiros. Esc. Anna Nery. 2009;13(1):145-53. 